\title{
DEUX PUBLICAINS ET LEUR TOMBEAU
}

\author{
Michel GAWLIKOWSKI
}

Lors des travaux de terrassement effectués à l'entrée ouest de la Vallée des Tombeaux pour y construire un barrage, une pierre inscrite a été découverte et aussitôt rapportée au musée par les responsables du chantier (fig. 1). Je tiens à exprimer tous mes remerciements à $\mathrm{M}$. Khaled As'ad, directeur des Antiquités et des Musées de Palmyre, pour avoir bien voulu autoriser la publication de ce monument.

Il semble que la pierre ait été enlevée jadis de son emplacement d'origine lors d'une opération de pillage et abandonnée au cours du transport. En effet, une dalle funéraire provenant manifestement du même tombeau aboutit dès 1889 au British Museum, comme on le verra tout à l'heure.

L'importance de l'inscription dépasse de loin l'intérêt onomastique que présentent d'habitude les textes de fondation des tombeaux palmyréniens. Elle apporte en effet de nouvelles données sur des personnages ayant rempli d'importantes fonctions dans la ville et qui sont connus par ailleurs.

L'inscription est gravée sur une plaque en calcaire dur, le champ épigraphique en retrait sur un cadre mouluré qui est conservé en bas et à droite; la partie haute de la plaque est cassée et la surface de la pierre abîmée du côté gauche. La hauteur conservée de la pierre s'élève à $65 \mathrm{~cm}$, la largeur à $108 \mathrm{~cm}, 1$ 'épaisseur à $25 \mathrm{~cm}$. L'inscription est trilingue; les lettres ont 7 et $5 \mathrm{~cm}$ pour le latin, $5 \mathrm{~cm}$ pour le grec, $2-3 \mathrm{~cm}$ pour le palmyrénien.

1. M. RODINSON, "Une inscription trilingue de Palmyre", Syria 27 (1950), p. 137; cf. aussi la trilingue honorifique pour le même personnage (Hairanem Bolnnae] qui et Rabbilum), de 74 ap. J.-C., où ses fonctions auprès du sanctuaire de Bel sont
[C. Virius Alcimus et]

[T. Stat]ILIUS HER[mes] [fec]ERUNT SIBI ET SUIS

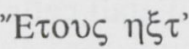

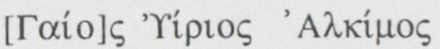

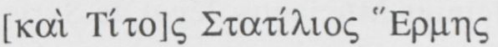

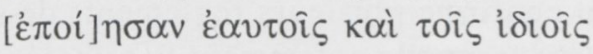
gys wyrs 'lqms wtyts 'stttlys $h$ [rms]

bnw npšh wm 'rth 'In lhn wlbnyhwn lyq[rhwn] bšnt 368

Je donne la traduction du texte palmyrénien, le plus complet, les noms propres retranscrits cependant en latin: "Gaïus Virius Alcimus et Titus Statilius Hermes ont construit ce monument funéraire et cet hypogée pour eux-mêmes et leurs enfants, en leur honneur, pour toujours, l'an 368 " (56/57 ap. J.-C.).

On connaît déjà deux autres trilingues funéraires, l'une et l'autre pratiquement contemporaines de notre inscription. Celle de Hairan, connu par ailleurs comme "décorateur des édifices des dieux", est de 52 ap. J.-C. ${ }^{1}$. L'autre concerne un percepteur des taxes (mks') du nom de L. Spedius Chrysanthus et date de 58 ap. J.-C. ${ }^{2}$.

Il est évident que ce trilinguisme dénote, selon le cas, des situations sociales différentes : autant Hairan, fils de Bônnâ, dit Rabbel, de la tribu des Benê Mîtâ, était palmyrénien de souche, sans doute devenu cosmopolite par contact avec l'architecte du

mentionnées : J. CANTINEAU, Syria 14 (1933), p. 174-176 (SEG VII, 1934, 133).

2. J. CANTINEAU, Inventaire VIII, 57 (CIS II 4235, IGRR III, 1539). 
temple de Bel et le personnel grec venu avec celui$\mathrm{ci}^{3}$, autant le percepteur Chrysanthus était étranger. Ce personnage, on le voit tout de suite, était l'affranchi d'un Romain, sans doute du publicain dont il était agent à Palmyre. Le cas d'Alcimus et d'Hermès est de toute évidence pareil à ce dernier : ils étaient tous les deux affranchis des citoyens romains, et leurs noms sont typiques du personnel servile d'origine grecque ou orientale.

On en resterait là si le nom d'Alcimus n'était mentionné dans le célèbre Tarif de 137 ap. J.-C., où l'on retrouve aussi le nom de Statilius, l'un et l'autre évoqués pour faire valoir un précédent relatif à la perception des taxes ${ }^{4}$. Il devient pratiquement certain que les deux associés l'étaient aussi dans l'exercice de leur profession, qui était celle de publicain.

L'édit de C. Licinius Mucianus, légat de Syrie de 67 à 69 , qui confirme plusieurs règlements de ses prédécesseurs ${ }^{5}$, rappelle notamment le principe de paiement en as italiques, exposé par "Germanicus César dans la lettre qu'il écrivit à Statilius" ( $\delta \imath \grave{\alpha}$ $\tau \hat{\jmath} \varsigma \pi \rho o ̀ \varsigma \Sigma \tau \alpha \tau \varepsilon i ́ \lambda \iota[o v \varepsilon \dot{\varepsilon} \pi \mathrm{l} \sigma] \tau o \lambda \hat{\eta} \varsigma)^{6}$; comme cette lettre remontait évidemment à l'époque de la mission de Germanicus en Orient, soit à 18/19 ap. J.-C., elle pourrait difficilement être adressée à Statilius Hermes de notre inscription de 56 ap. J.-C. Celui-ci était plutôt un affranchi d'un procurateur ou publicain correspondant de Germanicus; devenu avec le temps indépendant, il exerçait ses fonctions dans la même ville que jadis son patron.

Le nom d'Alcimus, en revanche, se rapporte selon toute vraisemblance au personnage même qui apparaît maintenant dans la nouvelle inscription. C'est en effet le nom personnel de cet affranchi, et

3. $C f$. E. WILL, dans H. SEYRIG, R. AMY, E. WILL, Le temple de Bel à Palmyre, Paris 1975, p. 215-223, et plus récemment, Les Palmyréniens. La Venise des sables, Paris, 1992, p. 134 s. Un cas parallèle est celui d'un maître tailleur Loukis Eras Zabou qui a laissé sa signature bilingue, avec un texte palmyrénien qui nomme ses collaborateurs locaux, sur un tambour du temple : Z. FIEMA, BASOR 263, 1986, p. 81-83.

4. Pour Statilius, CIS II 3913, IV 1,42; cf. PIR III, 588 (videtur procurator Caesaris in Syria a. 18 vel 19). Après R. CAGNAT, Revue de philologie 8 (1884), p. 141, et D. SCHLUMBERGER, Syria 18 (1937), p. 291, J.F. MATTHEWS, “The Tax Law of Palmyra”, JRS 74, 1984, p. 179, n. 30, pense que ce fonctionnaire pourrait être l'énigmatique tetagmenos du Tarif (CIS II 3913, gr. 130).

5. Texte rétabli par H. SEYRIG, "Le statut de Palmyre", non pas le gentilice, comme c'est le cas pour Statilius. Je n'ai pas retrouvé un Virius qui pourrait s'identifier au patron d'Alcimus.

Le passage correspondant du Tarif est très endommagé, au point que le contexte palmyrénien a été jugé inutilisable par J. Teixidor ${ }^{7}$, tandis que I.S. Shifman fournit un texte complété comme suit : "À propos des taxes, il y avait des controverses entre les Palmyréniens et le percepteur des taxes. J'ai décrété que [...] la taxe devrait être comme celle qu'a affermée en elle (sc. à Palmyre) Alcimus et son compagnon, selon la loi". Cette traduction (en russe chez Shifman) repose sur le texte palmyrénien restitué comme suit : ['l] ḩ̌bn $m k[s$ srbn hw'] byny tdmry' l[byny] / [s]q[w]t mksy[' ']qy[m]t dy ... [m]ks' / hyb lmhw' [hyk mk]s' [dy] 'gr bh / 'lqms wh [brh hyk] nmws'. Le grec est encore moins bien conservé : on y lit seulement $\mu \varepsilon \tau \alpha \xi i$ $\Pi \alpha \lambda[\mu \nu \rho \eta v \omega \hat{v} . .$.$] , ce qui correspond à byny tdmry'$ du texte palmyrénien ${ }^{8}$.

La première restitution de Shifman s'inspire d'un passage du préambule : srbnyn hww byny tgr' lbyny $m k s y^{\prime}, \zeta \eta \tau \eta \dot{\sigma} \sigma \iota \gamma^{\prime} \varepsilon^{\prime} v \varepsilon \sigma \theta[\alpha \imath \mu \varepsilon] \tau \alpha \xi \dot{v} \tau \omega \hat{v}$

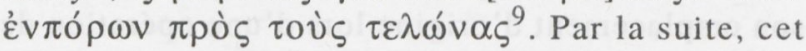
auteur supplée encore deux lectures hypothétiques. Celle de compagnon, $h b r h$, me paraît assez probable; cet associé d'Alcimus pourrait fort bien être identique à Statilius Hermes de notre inscription.

En revanche, je ne saurai admettre la restitution du mot $[s] q[w] t$, traduit comme "percepteur" d'après le syriaque sqotô qui à son tour procède du grec $\sigma \kappa v ́ \tau o \varsigma$, "fouet de cuir, courroie"10. Non seulement l'emploi syriaque est par trop métaphorique et l'on n'imagine pas comment il pourrait se généraliser, il faudrait encore admettre le passage du tet - en tav, phénomène dont Shifman cite quelques exemples, mais qui n'est pas courant. Cet

Syria 22 (1941), p. 165-167 (AS III, 152-154).

6. C'est sans doute le même Statilius qui apparaît aussi dans un contexte endommagé, contemporain de Germanicus et trouvé dans le sanctuaire de Bel : mn stltllys...Jlys, J. CANTINEAU, Syria 12 (1931), p. 139-141.

7. J. TEIXIDOR, "Palmyre, un port romain du désert", Semitica 34 (1984), p. 102 (palm. 11. 74-78); J.F. MATTHEWS, op. cit., p. 179 (traduction du grec); I. Sh. SHIFMAN, op. cit., p. 121 et 176-180.

8. I. Sh. SHIFMAN, op. cit., p. 102, I1. 25-26.

9. CIS II 3913

10. I. Sh. SHIFMAN, op. cit., p. 179 , contre H. INGHOLT, dans D. SChlumberger, "Réflexions sur la loi fiscale de Palmyre”, Syria 18 (1937), p. 289-290. 


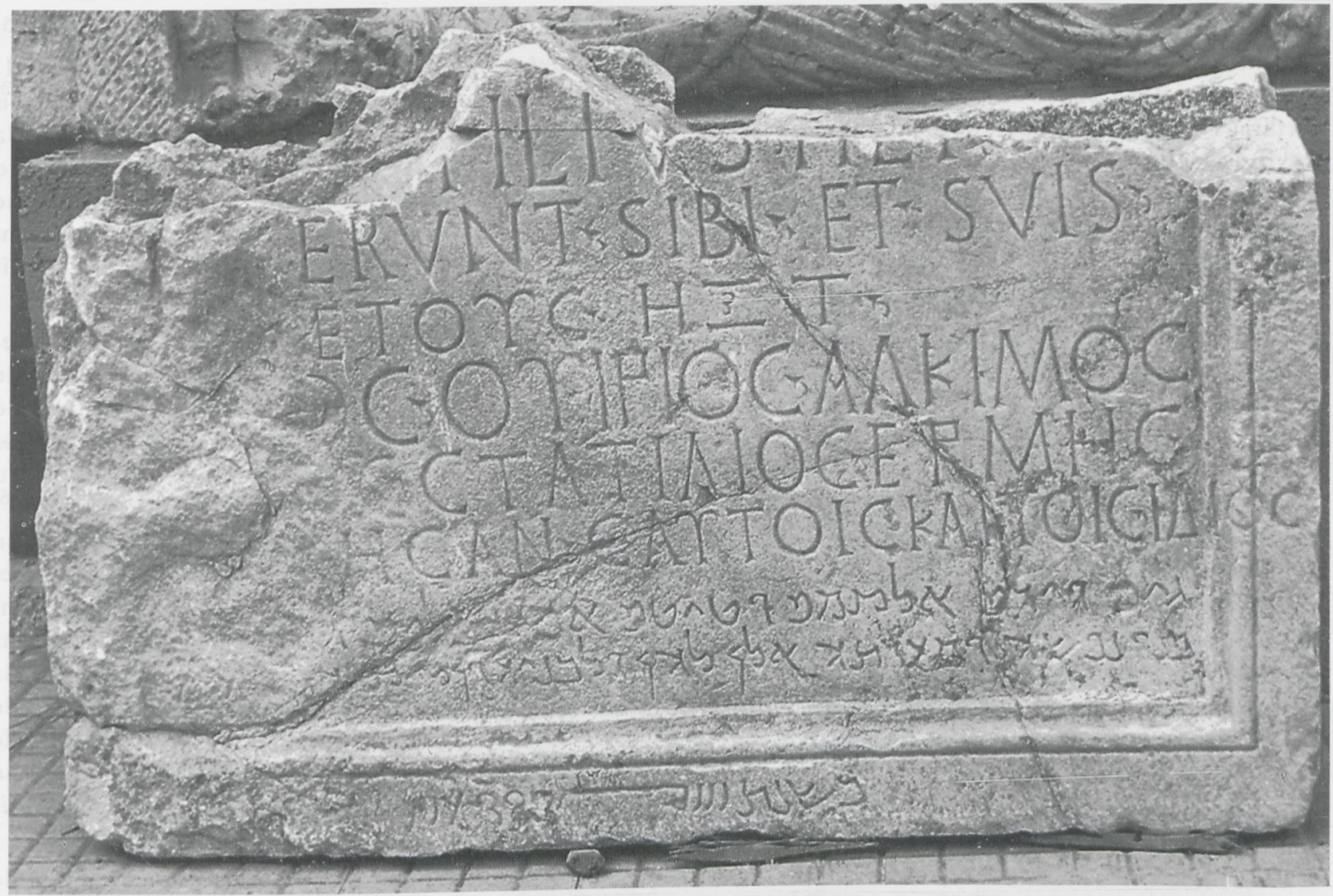

Fig. 1. - L'inscription du tombeau d'Alcimus et d'Hermès. Musée de Palmyre.

auteur rejette en même temps la lecture d'Ingholt et Schlumberger, ['l]qms mks [.], qui fournit au contraire un sens très satisfaisant : “... entre les Palmyréniens et Alcimus le percepteur”. J'ai vérifié cette lecture injustement ignorée sur la planche correspondante du Corpus, ainsi que sur la photo récemment publiée par J. Teixidor ${ }^{11}$. Il n'y a pas de doute qu'elle est correcte, et ce passage du Tarif se traduit donc comme suit (c'est Publicius Marcellus qui parle): "Parce que, quant aux taxes, [il y avait des différends] entre les Palmyréniens et [Al]cimus le publicain, j'ai décrété que la taxe [...] devra être [comme la ta]xe qu'avait affermée Alcimus et [son associé, selon] la loi"12.

Il demeurait jusqu'à présent incertain à quelle époque se situaient les activités d'Alcimus que l'on savait seulement antérieur à la légation de Mucien (67 à 69 ap. J.-C.), depuis qu'Henri Seyrig a rétabli ce dernier nom dans le texte. La nouvelle inscrip-

11. Aula Orientalis (Barcelone) 1, 1983, p. 244. tion montre que "Alcimus et son compagnon" étaient installés à Palmyre comme percepteurs déjà dans les années 50, et la nouvelle lecture du passage correspondant du Tarif permet de s'apercevoir que ce publicain restait toujours aux affaires sous le proconsulat de Mucien. Celui-ci confirmait simplement les modalités d'un contrat d'affermage conclu précédemment entre ce même Alcimus et la ville.

Les publicains de Palmyre affermaient, selon la coutume encore générale au $\mathrm{I}^{\mathrm{er}}$ siècle, les impôts indirects. C'est d'ailleurs le montant de ces impôts qui est 1'objet unique du Tarif. Cependant, selon l'interprétation - semble-t-il définitive - de ce document par H. Seyrig, ces taxes étaient en 137 ap. J.-C. régies souverainement par la Boulè, tandis qu'antérieurement elles étaient soumises au contrôle des autorités provinciales. Le contrat du publicain, et mêmes les détails de l'imposition,

12. Je comprends la préposition avec suffixe $b h$ comme complément d'objet indirect régi par le verbe ' $g r$, "affermer", se rapportant au nom $m k s$ ', "taxe" qui précède. 
avaient en effet relevé du gouverneur, dont l'administration y était sans doute directement intéressée. Bien entendu, rien n'empêchait que certaines dispositions anciennes soient maintenues par la nouvelle loi municipale.

Il est curieux de constater la présence simultanée à Palmyre des trois affranchis percepteurs. Autant il semble probable que deux d'entre eux formaient une compagnie de fermage d'impôts municipaux ("Alcimus et son associé", si la restitution du dernier mot par Shifman est correcte), autant les activités du troisième, Spedius Chrysanthus, ne s'inscrivaient nécessairement pas dans le même cadre. Était-il un fermier des douanes impériales, le "publicain du quart", comme sont appelés expressément ses collègues au $\mathrm{II}^{\mathrm{e}}$ siècle, Asclépiadès et Callistratus, et comme le suppose déjà F. Millar dans son ouvrage récent ${ }^{13}$ ? C'est probable, mais plus frappant encore est le fait que les taxes municipales sont, celles-ci de façon certaine, affermées dans tous les cas connus à des affranchis des Romains, étrangers à la cité; outre Statilius Hermes et Virius Alcimus, actifs dans les années 50, on peut citer encore l'affranchi impérial Cilix, mentionné par le Tarif, de date indéterminée. Leur dépendance vis-à-vis des autorités romaines va de soi.

Il en allait autrement au début du siècle : en 10/ 11 ap. J.-C., le percepteur du "péage des chameaux" répondait au nom de "Atenatan, appartenait à l'une des tribus principales de l'oasis et n'avait des comptes à rendre que devant "le peuple des Palmyréniens" ${ }^{14}$. Ce que le régime de Palmyre a changé entre-temps : la cité a été annexée à la province, peut-être par Germanicus, mais plus probablement quelques années plus tôt, lorsque le gouverneur Creticus Silanus (12-17 ap. J.-C.)

13. Inv. X, 29 et 113; $f$. F. MILlAR, The Roman Near East, Cambridge, Mass.-London 1993, p. 324.

14. M. GAWLIKOWSKI et Kh. AS‘AD, "Le péage à Palmyre en 11 ap. J.-C.", Semitica 41-42 (1993), p. 163-172. Je ne suis pas convaincu par l'interprétation de ce texte par B. AGGOULA, Syria 71 (1994), p. $415-417$ ("sépultures des chameliers") ni surtout pas ses conclusions générales.

15. Pour la borne de Khirbet Bil'as, publiée par D. SCHLUMBERGER, Syria 20 (1939), p. 43 s., $c f$. J.-Ch. BALTY, ANRW II, 8 (1977), p. 118 s. et, en dernier lieu J.F. MATTHEWS, op. cit, p. 162 : a]rva civitat[is Apam]enorum, plutôt que [Hemes]enorum.

16. $C f$. M. GAWLIKOWSKI, Le temple palmyrénien, Varsovie 1973, p. 41-45; pour l'annexion, voir maintenant E. WILL, Les Palmyréniens. La Venise des sables, Paris 1992, p. 39-46 et F. MILlAR, op. cit., p. $324 \mathrm{~s}$; ; contre la thèse traditionnelle de aborna le territoire de Palmyre du côté d'Apamée ${ }^{15}$. En tout cas, l'incorporation eut lieu après l'an 11, date de l'inscription récemment découverte du percepteur "Atenatan, sans que les institutions de la cité aient été immédiatement modifiées: "le peuple" est encore attesté en 51 ap. J.-C., alors que la Boulè, conforme au modèle hellénistique courant et favorisé par le gouvernement romain, n'apparaît que dans une inscription de 74 ap. J.-C.

Une circonstance heureuse permet de rejoindre, par ces considérations historiques, les questions de l'art palmyrénien. Le hasard a voulu que, peu de temps après avoir lu l'inscription d'Alcimus et d'Hermès, je suis tombé, lors d'une visite au British Museum, sur la pierre tombale du premier ${ }^{16}$. Comme l'inscription est rédigée exclusivement en grec, elle ne figure naturellement pas dans le CIS et semble avoir échappé à tous ceux qui s'occupaient du Tarif palmyrénien. La sculpture a été notée, sans plus, par les ouvrages de référence, sans avoir réveillé beaucoup d'intérêt ${ }^{17}$. Je reproduis la belle photo qui m'a fort obligeamment été fournie par le musée, grâce à l'amabilité de Mme Dominique Collon, conservateur des Western Asiatic Antiquities (fig. 2).

Il s'agit d'une dalle en calcaire dur ${ }^{18}$, haute de $70 \mathrm{~cm}$, représentant un couple en buste au-dessus d'une plinthe qui porte en creux le champ épigraphique entouré d'une simple moulure en doucine. Le fond derrière les personnages, sommairement dégrossi, est cintré ${ }^{19}$. L'inscription donne les noms des époux, chacun inscrit dans une moitié correspondante du champ, séparé par une ligne verticale :

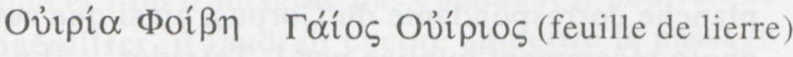
'A $\lambda$ кí $\mu$ os

H. SEYRIG (supra, n. 5) sur Palmyre ville libre, $c f$. J. TEIXIDOR, op. cit., p. 11 et 91 s., et maintenant F. MILLAR, loc. cit.

17. H. INGHOLT, Studier over Palmyrensk Skulptur, Copenhagen 1928, p. 94, PS 60 (mention seulement); M.A.R. COLledge, The Art of Palmyra, London 1976, p. 69. fig. 76 .

18. Numéro du musée WA 125036, numéro d'entrée 89-1012,1 , ce qui indique l'acquisition en 1889 , comme me le signale obligeamment K. Parlasca.

19. Cette forme rare pour une plaque de loculus se retrouve deux fois dans le tombeau d'Artaban: A. SADURSKA et A. BOUNNI, Les sculptures funéraires de Palmyre, Rome 1994, p. 33-34, nos 33-34, fig. 25, 136; cf. K. TANABE, Sculptures of Palmyre I, Tokyo 1986, pl. 233. Les deux monuments sont datés entre 100 et 130 ap. J.-C. d'après leur style. 


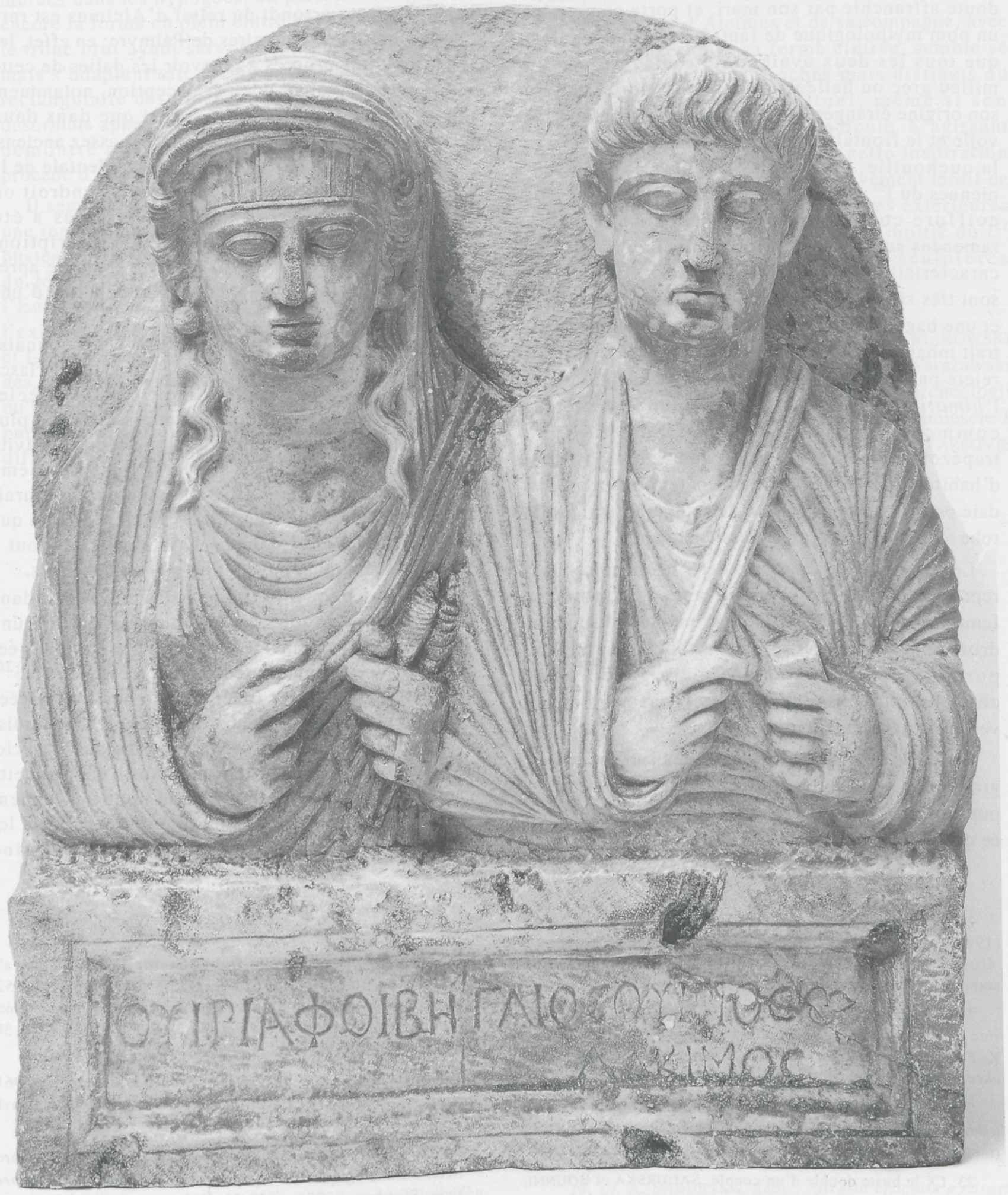

Fig. 2. - Pierre tombale d'Alcimus et Phoebè. British Museum. 
On voit tout de suite que l'homme porte le même prénom et gentilice que notre Alcimus et qu'il lui est donc identique ${ }^{20}$. Sa femme Phoebè a été sans doute affranchie par son mari, et porte comme lui un nom mythologique de fantaisie, indiquant ainsi que tous les deux avaient été esclaves dans un milieu grec ou hellénisé. La femme porte, malgré son origine étrangère, le costume local type, avec le voile et le frontal ${ }^{21}$, tient de sa gauche le fuseau et la quenouille, attributs constants des Palmyréniennes du $\mathrm{I}^{\mathrm{er}}$ et jusqu' au milieu du $\mathrm{II}^{\mathrm{e}}$ siècle, et sa coiffure comporte deux mèches de cheveux ramenées sur le buste, selon la manière non moins caractéristique de la même époque ${ }^{22}$. Ses bijoux sont très simples : des boucles d'oreille globulaires et une bague indistincte sur l'index gauche. Le seul trait inhabituel consiste en la façon dont le voile est rejeté par-dessus l'épaule gauche, à la manière de l'himation masculin. Il semble que le sculpteur commençat d'indiquer une fibule de type trapézoïdal, retenant le voile dans cette position; d'habitude, la fibule des femmes, toujours trapézoïdale pour le premier groupe de sculpture, agrafait la robe au même endroit par-dessous le voile.

Le portrait d'Alcimus est des plus banals. Il est représenté légèrement vers l'avant par rapport à sa femme et porte un himation qui enveloppe son bras droit pour passer sur l'épaule gauche. L'homme porte une tablette dans sa main gauche. Ses cheveux sont ramenés sur le front en mèches verticales légèrement ondulées ${ }^{23}$.

Les visages sont traités en larges surfaces assez grossièrement définies, les yeux vides (peut-être les pupilles étaient-elles peintes à l'origine). Sauf pour ce dernier détail, le relief est un exemple type de la

20. L'inscription a été commentée par K. PARLASCA, DaM 3 (1988), p. 218, qui voulait corriger le gentilice en Ovi $\beta \iota \alpha /$ Ovíßtos; notre nouvelle inscription montre que le patron commun de ces affranchis s'appelait bien Virius.

21. Comme plus tard, cette autre affranchie Annia Nice, nourrice de Baebia Baebiana (lire ainsi, malgré H. HEINEN, dans : K. PARLASCA, Syrische Grabreliefs hellenistischer und römischer Zeit, Mainz/Rh. 1981, p. 35-38, pl. 24,4).

22. Cf. SADURSKA et BOUNNI, op. cit., p. 18, fig. $133, \mathrm{n}^{\circ} 10$ (tombeau de 'Aštor, après 90); p. 32-33, fig. 135-136, n 32-33 (tombeau d'Artaban, début $\mathrm{II}^{\mathrm{e}}$ siècle).

23. $C f$. le buste double d'un couple, SADURSKA et BOUNNI, op. cit., p. 62 , fig. $20, \mathrm{n}^{\circ} 80$ (= TANABE, pl. 368 ), daté au tournant du $\mathrm{I}^{\mathrm{cr}}$ siècle.

24. M. GAWLIKOWSKI, Monuments funéraires de Palmyre, première catégorie de la sculpture palmyrénienne comme définie par $\mathrm{H}$. Ingholt, antérieure à $150 \mathrm{ap}$. J.-C. environ.

La contour arrondi du relief d'Alcimus est rare parmi les bustes funéraires de Palmyre; en effet, les loculi cintrés propres à recevoir les dalles de cette forme ne s'y voient que par exception, notamment dans l'hypogée d'Artaban, ainsi que dans deux monuments hors série, qui paraissent assez anciens, situés tous les deux à l'extrémité occidentale de la Vallée des Tombeaux, non loin de l'endroit où l'inscription d'Alcimus et de Hermès a été retrouvée ${ }^{24}$. De même, la plinthe à inscription, courante ailleurs, ne se voit guère à Palmyre après le $\mathrm{I}^{\mathrm{er}}$ siècle, sinon sur le monument déjà cité d'une autre affranchie étrangère ${ }^{25}$.

Le style de la sculpture suggéra à des connaisseurs tels que Malcolm Colledge ou Klaus Parlasca une date dans la première moitié du II $^{\mathrm{e}}$ siècle. Cependant, notre sculpture est nécessairement plus ancienne. Comme Alcimus était en pleine activité entre 57 et 67 ap. J.-C., sa pierre tombale, même admettant que dédiée à titre posthume, ne saurait dépasser le dernier quart du $\mathrm{I}^{\mathrm{er}}$ siècle. On a vu que les parallèles du tombeau d'Artaban cités tout à l'heure se regroupent vers l'an 100 ou peu après.

À cette époque, le tombeau appelé napšâ dans l'inscription de fondation ne pourrait être qu'une tour funéraire, dans ce cas munie d'un hypogée, comme on en connaît précisément à cette époque ${ }^{26}$. On sait que les stèles funéraires en buste, destinées justement en premier lieu à des hypogées, apparaissent à Palmyre dans la seconde moitié du $\mathrm{I}^{\mathrm{er}}$ siècle, le premier exemple daté remontant à $65 / 66$; cette série, promise à une popularité dont témoignent toutes les collections, remplace progressivement les stèles en pied ${ }^{27}$. Celles-ci étaient à l'origine

Varsovie 1970, p. 66-67.

25. K. PARLASCA, "Ein frühes Grabrelief aus Palmyra", Eikones (Festschrift Hans Jucker), Bern 1980, p. 149-152, pl. 50,1 et 51,$2 ; c f$. H. SEYRIG, "Notes sur les plus anciennes sculptures palmyréniennes”, Berytus 3 (1936), p. 137 s., pl. 30. Pour l'affranchie, supra, note 21.

26. K. MICHALOWSKI, Palmyre fouilles polonaises 1961, Varsovie 1963, p. 197 s.; M. GAWLIKOWSKI, op. cit., Varsovie 1970 , p. $60-68$.

27. Cf. K. PARlaSCA, "Probleme palmyrenischer Grabreliefs. Chronologie und Interpretation", Palmyre bilan et perspectives, Strasbourg 1976, p. 33-43; H. INGHOLT, dans : Mélanges Michalowski, Varsovie 1966, p. 459-463, fig. 2-4; pour le buste de 65/66, F.O. HVIDBERG-HANSEN, G. Ploug, Palmyra Samlingen, Ny Carlsberg Glyptotek 1993, p. 42. 
connues pour marquer les sépultures en terre et présentent d'habitude un champ cintré entouré d'un listel. Vers la fin du $\mathrm{I}^{\mathrm{er}}$ siècle, on les retrouve murées dans les hypogées, où parfois elles gardent encore la forme en plein cintre et conservent même le tronc brut ayant servi à les planter dans le sol, mais s'adaptent vite à l'ouverture habituellement rectangulaire des niches mortuaires qu'elles sont désormais appelées à fermer. Il n'est pas cependant démontré que le buste funéraire palmyrénien procède d'une "évolution" de ces stèles.

Il semble au contraire qu'il y a lieu de supposer une inspiration extérieure. En effet, un groupe des bustes funéraires provenant de Belkis et peut-être de Membidj (dans l'Antiquité, Zeugma-surl'Euphrate et Hiérapolis) ${ }^{28}$ permet de concevoir l'existence d'une source commune pour cette série et la série de Palmyre. Ces sculptures présentent des bustes ou demi-figures dans des niches cintrées ou rectangulaires et sont habituellement identifiées par les inscriptions en grec sur la plinthe. Assez différente par son style de l'art palmyrénien mais

28. J. WAGNER, Seleukeia am Euphrat/Zeugma, Wiesbaden 1976. Cf. K. PARLASCA, Syrische Grabreliefs hellenistischer und römischer Zeit, Mainz/Rh. 1981, p. 9-14. proche de lui par l'iconographie, la série commence au $\mathrm{I}^{\text {er }}$ siècle et continue jusqu' au III $^{\mathrm{e}}$; elle est donc parallèle à celle, beaucoup plus fournie, de Palmyre ${ }^{29}$.

Le bas-relief d'Alcimus et de sa compagne, avec sa plinthe inscrite et sa forme cintrée, semble se référer à des modèles proches mais distincts du schéma palmyrénien habituel, même si son exécution locale ne fait pas de doute. S'agissant d'un couple étranger à l'oasis, cette inspiration explique à mon sens 1 'aspect relativement tardif de cette sculpture que les circonstances extérieures placent fermement dans la seconde moitié du $I^{\text {er }}$ siècle, parmi les plus anciennes sculptures funéraires palmyréniennes.

M. GAWLIKOWSKI Uniwersytet Warszawski Instytut archeologii Krakowskie Przedmiescie 1 00.047 VARSOVIE
29. $C f$. A. SADURSKA, dans : Ritratto ufficiale e ritratto privato (Atti della II Conferenza Internazionale sul Ritratto Romano, 1984), Rome 1988, p. 83 s. 